\title{
Stokes-Einstein relation of the liquid metal rubidium and its relationship to changes in the microscopic dynamics with increasing temperature
}

\author{
F. Demmel ${ }^{1, *}$ and A. Tani ${ }^{2, \dagger}$ \\ ${ }^{1}$ ISIS Facility, Rutherford Appleton Laboratory, Didcot OX11 0QX, United Kingdom \\ ${ }^{2}$ Dipartimento di Chimica, Universita di Pisa, Via G. Moruzzi 13, I-56124 Pisa, Italy
}

(Received 27 February 2018; published 12 June 2018)

\begin{abstract}
For liquid rubidium the Stokes-Einstein (SE) relation is well fulfilled near the melting point with an effective hydrodynamic diameter, which agrees well with a value from structural investigations. A wealth of thermodynamic and microscopic data exists for a wide range of temperatures for liquid rubidium and hence it represents a good test bed to challenge the SE relation with rising temperature from an experimental point of view. We performed classical molecular dynamics simulations to complement the existing experimental data using a pseudopotential, which describes perfectly the structure and dynamics of liquid rubidium. The derived SE relation from combining experimental shear viscosity data with simulated diffusion coefficients reveals a weak violation at about $1.3 T_{\text {melting }} \approx 400 \mathrm{~K}$. The microscopic relaxation dynamics on nearest neighbor distances from neutron spectroscopy demonstrate distinct changes in the amplitude with rising temperature. The derived average relaxation time for density fluctuations on this length scale shows a non-Arrhenius behavior, with a slope change around $1.5 T_{\text {melting }} \approx 450 \mathrm{~K}$. Combining the simulated macroscopic self-diffusion coefficient with that microscopic average relaxation time, a distinct violation of the SE relation in the same temperature range can be demonstrated. One can conclude that the changes in the collective dynamics, a mirror of the correlated movements of the particles, are at the origin for the violation of the SE relation. The changes in the dynamics can be understood as a transition from a more viscous liquid metal to a more fluid-like liquid above the crossover temperature range of 1.3-1.5 $T_{\text {melting. }}$ The decay of the amplitude of density fluctuations in liquid aluminium, lead, and rubidium demonstrates a remarkable agreement and points to a universal thermal crossover in the dynamics of liquid metals.
\end{abstract}

DOI: 10.1103/PhysRevE.97.062124

\section{INTRODUCTION}

A liquid has borders to the gas phase at the boiling point and to the solid state at the melting point. Crossing the boiling point the main change is a decrease in density by several orders of magnitude. Freezing a liquid into a crystal the density changes only in the percent level; however, long-range order sets in and breaks the rotational symmetry of the liquid state. If crystallization can be avoided the liquid can be undercooled and eventually the material morphs into a glass with dramatic consequences for transport parameters like diffusion and viscosity [1]. Less dramatic are the changes in the dense equilibrium liquid phase, which is characterized by disorder, strong particle correlations, and a vanishing resistance against shear forces, expressed in a vanishing static shear modulus of the liquid. The last statement has to be amended when the changes occur faster than typical relaxation times of the liquid.

The Stokes-Einstein (SE) relation connects the shear viscosity of a solvent with the diffusion coefficient of a large particle immersed in it. Einstein related the diffusive movements of a Brownian particle in the solvent to the frictional force of the surrounding solvent through Stokes' law [2]. That relation is based on hydrodynamics to describe the drag by a solvent with

\footnotetext{
*franz.demmel@stfc.ac.uk

†alessandro.tani@unipi.it
}

shear viscosity $\eta$ on a diffusing spherical particle [3]:

$$
D=\frac{k_{B} T}{C \eta \sigma},
$$

where $D$ is the diffusion constant, $k_{B}$ the Boltzmann constant, $T$ the temperature, and $\sigma$ the diameter of the moving particle. $C$ is a constant which depends on the interface conditions between particle and solvent. It is $2 \pi$ for slip conditions and $3 \pi$ for nonslip conditions [4]. Early on some criticism has been put forward that this relation describing a Brownian particle in a solvent is only an approximation for very slowly moving particles [5]. However, it turned out that the SE relation works reasonably well even when the particle has the same size and mass as the solvent particle, hence for atomic systems. As there is some ambiguity with the so-called molecular diameter [6], $\sigma$ may be and has been regarded as an effective hydrodynamic diameter. For example, for liquid rubidium with a shear viscosity of $\eta=55 \times 10^{-5} \mathrm{~Pa} \mathrm{~s}$ [7] at $T=320 \mathrm{~K}$ and a particle diameter of $\sigma=4.44 \AA$ [8] we arrive at a diffusion coefficient of $D=2.88 \times 10^{-5} \mathrm{~cm}^{2} / \mathrm{s}$ for the slip condition. An experimental value for the diffusion coefficient near the melting temperature $\left(T_{\text {melting }}=312.65 \mathrm{~K}\right)$ of $D=$ $2.7 \times 10^{-5} \mathrm{~cm}^{2} / \mathrm{s}$ was reported [9]. That good agreement supports the widespread opinion that the SE relation is well fulfilled even for monatomic liquids.

Despite the good agreement in many cases extensions of the simple SE relation have been proposed. Zwanzig proposed a variant of the SE relation which included longitudinal 
viscosity [10] and generalizations based on frequencydependent friction coefficients [11]. Balucani et al. proposed a wave-vector-dependent shear viscosity $\eta(q)$ for a generalized SE relation, which improved the agreement between calculation and experiment in the case of liquid sodium $[12,13]$. To add more options further representations for the SE relation are used, based on replacing the viscosity by a relaxation time [14].

The shear viscosity $\eta$ can be represented applying the stress tensor autocorrelation function $[4,15]$. At shorter wavelengths a $Q$-dependent viscosity can be introduced. Within a viscoelastic response of the fluid a wave-vector- and time-dependent viscosity is defined, which describes the decay of shear fluctuations. The wave-vector-dependent Maxwell relaxation time $\tau(Q)$ is the decay constant for these relaxations and intimately related to the viscosity: $\eta(Q)=\tau(Q) G_{\infty}(Q)$ with a high-frequency shear modulus $G_{\infty}(Q)$ [13]. That relation is the basis to replace the viscosity with a relaxation time in the SE relation: $D \tau \propto T$. Underlying is the assumption that the high-frequency shear modulus $G_{\infty}(Q)$ is temperature independent, which is in a small enough temperature range certainly well fulfilled.

The main interest in the most recent reports on the SE relation concerns less the absolute value rather than the evolution with temperature and potential violations of the relation. The SE relation can be written as

$$
D \eta \propto T \text { or } D \tau \propto T .
$$

Both forms have extensively been used to follow changes in the dynamics, in particular, towards lower temperatures. Shi et al. discuss different variants of the SE relation and using MD simulation data they demonstrate different behavior with temperature change [14]. One of the observations was that the linear proportionality to temperature might be replaced by a fractional dependence, coined the fractional SE relation [16-18]. Furthermore, it has been observed that liquids in the supercooled state demonstrate a breakdown of the SE relation toward the glass transition $[19,20]$. That breakdown is related to a decoupling in dynamics and was regarded as a sign of dynamic heterogeneity. It should be kept in mind that self-diffusion and viscosity are based on different statistical averages: self-diffusion averages over the movement of a tagged particle, and viscosity takes into account the correlations of different particles. Therefore a departure of these two different dynamical processes might be expected at certain thermodynamic conditions.

Interestingly, reports have been made that the breakdown of the SE relation can occur above the liquidus temperature deep in the liquid state. For a metallic alloy experimental data were reported that the product of the measured macroscopic viscosity and microscopic diffusivity are independent of temperature above the liquidus temperature over a range of about $400 \mathrm{~K}$ [21]. MD simulations on CuZr alloys demonstrated the breakdown of the SE relation about $500 \mathrm{~K}$ above the derived melting point of the alloy $[22,23]$. Classical MD simulations on a CuZrAl alloy revealed a breakdown of the SE relation above the melting point [24] and ab initio simulations on $\mathrm{Al}-\mathrm{Cu}$ alloys also reported a breakdown of the SE relation above the liquidus temperature [25]. These results point to a profound change in the dynamics within the liquid state of the melts.
On a microscopic length scale within the equilibrium liquid state changes in the relaxation dynamics with increasing temperature have been observed for several liquid metals, like rubidium, lead, and aluminium [26-28]. These studies demonstrate distinct changes in the dynamics of density fluctuations on atomic length scales with increasing temperature from the melting point up to about twice the melting temperature. For rubidium an additional slow relaxation process has been identified by MD simulation and explained through mode-coupling theory [29]. That slow process was related to structural freezing and was predicted to increase strongly in the supercooled state. Sophisticated neutron scattering experiments proved the existence of this slow relaxation process near the melting point [30] and a later investigation showed that this slow process disappears at about $T \approx 400 \mathrm{~K} \approx 1.3 T_{\text {melting [31]. }}$ Furthermore, the amplitude $S(Q, \omega=0)$ is interrelated to a generalized longitudinal viscosity coefficient $\eta_{l}(Q)$. The longitudinal viscosity is a measure for the diffusion of momentum parallel to the velocity of the particles and can be regarded as being sensitive to atomic-level stress correlations, in our case, on next neighbor distances. The evaluation of $\eta_{l}(Q)$ showed a steplike increase of the generalized viscosity with decreasing temperature at around $400 \mathrm{~K}$ [26]. In addition, MD simulations of generalized viscosities at the structure factor maximum confirmed that increase with decreasing temperature [32]. The increase in generalized viscosity and the increase of the amplitude $S\left(Q_{0}, \omega=0\right)$, related to the set-in of an additional slow relaxation process, are evidence for a change in the dynamics to a more viscous, solid-like state upon cooling at around $400-450 \mathrm{~K}$, in a temperature range of about $1.3 T_{\text {melting }}$. These pieces of evidence for a change in liquid dynamics on an atomic length scale provoke the question of whether changes exist in thermodynamic and transport coefficients in the same temperature range.

Many studies on violations of the SE relation and changes in liquid dynamics are based on MD simulations. However, for liquid rubidium a wealth of experimental data of macroscopic transport parameters exists [7,9]. Rubidium has a suitable coherent cross section for neutrons and only a negligible incoherent scattering cross section [33], which provided perfect conditions to study collective dynamics and in particular the coherent excitations in a simple liquid metal in detail over the past decades; see for example [34-39]. Collective data have been obtained over a wide range of temperatures, yet mostly in coarse temperature steps. The observation of welldefined inelastic excitations over a wide range in wave numbers initiated theoretical investigations based on mode coupling theory $[40,41]$. Due to the neutron scattering properties of the rubidium nucleus no self-dynamics experimental data are available and simulations are the only source for this information. The availability of a reliable pseudopotential, which described structure and then dynamics quite well, prompted many classical MD simulation studies; see for example [4247]. Later on first-principles methods have also been applied to simulate liquid rubidium [48-50].

Here we present changes in macroscopic transport parameters of liquid rubidium cast in the SE relation over a temperature range up to twice the melting temperature. We complement the available experimental shear viscosity coefficients with data from a classical molecular dynamics (MD) simulation, 
which provides access to the not experimentally available selfdynamics. The average relaxation time of density fluctuations at next neighbor distances is obtained in small temperature steps from neutron scattering and is then connected with the simulated self-diffusion coefficient. This relation highlights the importance of correlated particle movements when changes in the dynamics are discussed.

\section{COMPUTATIONAL AND EXPERIMENTAL DETAILS}

We carried out a set of MD simulations to complement the neutron scattering results. Structural and dynamical properties of liquid alkali metals have been successfully predicted via classical MD simulations using effective potentials [51-53]. As in our earlier study of liquid rubidium [32], we adopt the widely used Price potential $[42,53,54]$. Price's potential is an effective, density-dependent potential based on a local pseudopotential [55], which describes the bare electron-ion interaction [56], and a dielectric function $d(k)$, which describes conduction-electron screening [57]. The potential has the following form:

$$
v(r)=\frac{(Z e)^{2}}{r}\left[1-\frac{2}{\pi} \int_{0}^{\infty} d k \frac{\sin (k r)}{k} \cos ^{2}\left(k r_{c}\right) \frac{d(k)-1}{d(k)}\right],
$$

where $Z e$ is the ion charge, and $r_{c}=2.40 \AA$ represents the radius of the ion core. For $d(k)$ we use the form described by Price et al. [54]. Comparing with the more common Lennard-Jones potential, Price's potential shows small oscillations; it is softer and short ranged. These small differences produce substantial changes in the propagation of the density fluctuations in the liquid at wavelengths comparable to the inter-ion distance. The parameters that characterize the potential are the particle density $(\rho)$, the first zero of the potential $(\sigma)$, and the depth of the potential $(\epsilon)$. The system size was increased eight times with respect to the previous paper [32] to a number of 6912 atoms. The cubic box size has been adjusted to the experimental density, which was kept fixed, so the simulated system belongs to the microcanonical ensemble. The equation of motion of all particles were integrated using the Verlet algorithm with an integration step $\delta t=5$ fs. The potential was set to zero for distances greater than $r_{\text {cut }}=5.5 \sigma$. This large cutoff distance and the fine time step allowed a quite satisfactory energy conservation, even in rather long runs. Equilibration took at least $1 \mathrm{~ns}$ and up to $2 \mathrm{~ns}$ at the lower temperatures. Averages were taken over multiple runs, each $0.5 \mathrm{~ns}$ long at each temperature. The number of runs ranged from 4 at $T=600 \mathrm{~K}$ to 12 at $315 \mathrm{~K}$. We analyzed the static and dynamical properties of liquid rubidium at ten different temperatures starting from the supercooled region $T=280$ to $600 \mathrm{~K}$, almost twice as large as the melting point. A homemade software package was used for the simulations, which has been continuously extended over the past decades.

We chose the wave vectors $\mathbf{Q}=\left(Q_{x}, Q_{y}, Q_{z}\right)$ such that $Q_{i}=n_{i} \frac{2 \pi}{L}, i=(x, y, z)$ with $n_{i}$ integer. The system size allowed us to reach a minimum wavelength of roughly $0.07 \AA^{-1}$, well in the hydrodynamic regime. For a given wave vector $Q$ the physical properties calculated during the simulations are averages on all the possible orientations of $Q$. For each temperature we can evaluate structural and dynamical properties. Directly from the MD simulations, at each time $t$, we obtain the positions of each rubidium particle $\left[\mathbf{r}_{j}(t), j=\right.$ $1 \ldots N]$. Knowing the time evolution of the configurations, we evaluate $F(Q, t)$ as a correlation function of the space Fourier transform of the density $n(Q, t)$ :

$$
F(Q, t)=\left\langle n^{*}(\mathbf{Q}, \tau) n(\mathbf{Q}, t+\tau)\right\rangle,
$$

where

$$
n(Q, t)=\frac{1}{\sqrt{N}} \sum_{j=1}^{N} e^{i \mathbf{Q} \cdot \bar{r}_{j}(t)} ;
$$

$\langle\cdots\rangle$ indicates the average over many different initial conditions $\tau$ and over many different $\mathbf{Q}$ vectors of magnitude $Q$. We obtain the intermediate scattering function $F(Q, t)$, from which we determine its spectrum $S(Q, \omega)$, the dynamical structure factor. The same procedure applies to the self-dynamics.

On a microscopic length scale the diffusion coefficient $D$ is related through a Green-Kubo relation with the velocity autocorrelation function $Z(t)=\frac{1}{3}\langle\mathbf{v}(t) \cdot \mathbf{v}(0)\rangle$ of a tagged particle [4]:

$$
D=\frac{1}{3} \int_{0}^{\infty}\langle\mathbf{v}(t) \cdot \mathbf{v}(0)\rangle d t=\int_{0}^{\infty} Z(t) d t .
$$

The initial value is $Z(0)=\frac{k_{B} T}{m}$ with $k_{B}$ the Boltzmann constant, $T$ the temperature, and $m$ the mass of the particle. The diffusion coefficient can also be determined from the long-time limit of the mean-squared displacement of a tagged particle:

$$
D=\lim _{t \rightarrow \infty} \frac{\left\langle\left|r_{i}(t)-r_{i}(0)\right|^{2}\right\rangle}{6 t}
$$

We actually exploited both methods, also averaging over the three independent components of displacement and velocity. The $D$ values agree within $1 \%$, which can be regarded as the measure for uncertainty.

To demonstrate the good agreement between the modeled rubidium and experimental data we show in Fig. 1 the structure factor of liquid rubidium near the melting point from x-ray

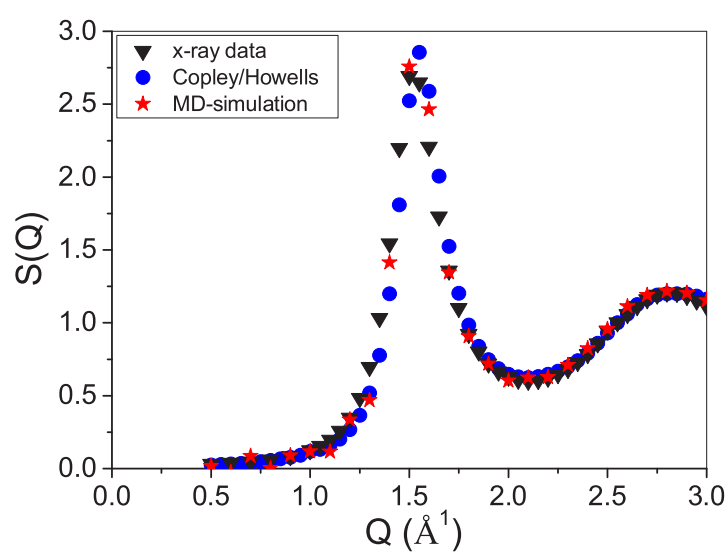

FIG. 1. A comparison between the experimental and the simulated structure factor $S(Q)$ near the melting point is presented. 


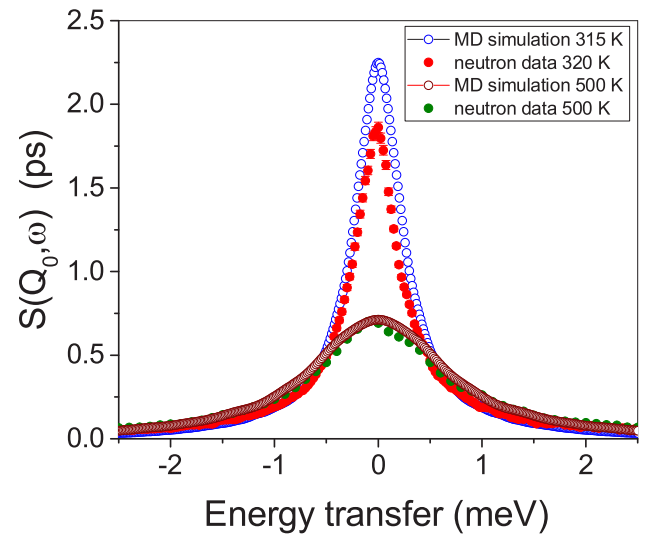

FIG. 2. Experimental spectra $S\left(Q_{0}=1.5 \AA^{-1}, \omega\right)$ (full symbols) and the spectra from the MD simulation (open symbols) demonstrate the high quality of agreement between experiment and simulation on an absolute intensity scale.

scattering, neutron scattering [7], and our simulations. There is a nearly perfect agreement concerning the structure. Figure 2 shows $S\left(Q_{0}, \omega\right)$ spectra at $320 \mathrm{~K}$ and $500 \mathrm{~K}$ from neutron data [31] compared with the simulated spectra of the dynamic structure factor. These are absolute normalized spectra without any scaling factor between them. The widths of both spectra are practically identical; however, the amplitude of the simulated spectrum at low temperature is slightly larger. There might be some averaging in the measured spectrum due to a necessary integration in momentum space, which might explain the differences in the height of the spectra near the melting point. At higher temperature the spectra amplitude agree perfectly, probably because the structure factor peak is already not so sharp anymore.

The coherent dynamic structure factor $S(Q, \omega)$ of liquid rubidium has been measured by neutron scattering. Rubidium is a nearly coherent scatterer with an incoherent contribution of less than 0.04 barns compared to the coherent cross section of 6.24 barns [33]. Three experiments with differing energy resolutions have been performed to follow the changes in dynamics at the structure factor maximum. A first series of experiments has been performed at the three-axis spectrometer with the multi-analyzer-detector unit of the Forschungsreaktor München (FRM) [58]. Constant- $Q$ scans at thirteen temperatures have been measured with an energy resolution of $\mathrm{FWHM}=1.3 \mathrm{meV}$. A second series of experiments has been performed at the thermal three-axis spectrometer IN3 at the Institute Laue Langevin, Grenoble. At a final energy of $14.66 \mathrm{meV}$ an energy resolution of FWHM $=0.8 \mathrm{meV}$ was obtained. A third series of experiments was undertaken at the IRIS spectrometer of the ISIS Facility. A configuration with an end energy of $7.38 \mathrm{meV}$ was chosen, which provided an excellent energy resolution of FWHM $=0.055 \mathrm{meV}$. More experimental details, also about multiple scattering corrections, can be found in $[26,31]$. Figure 3 illustrates the change of the spectral amplitude with rising temperature. From $320 \mathrm{~K}$ to 400 $\mathrm{K}$ the amplitude changes about twice as much as between 400 $\mathrm{K}$ and $550 \mathrm{~K}$, indicating a change in relaxation dynamics at next neighbor distances with rising temperature.

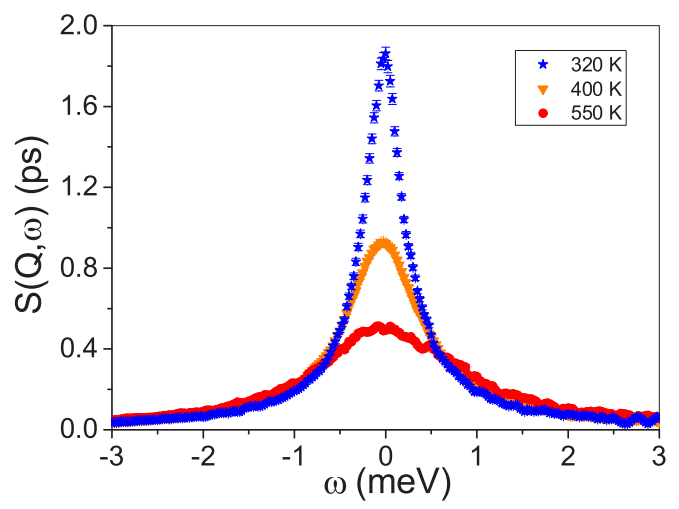

FIG. 3. Three experimental spectra $S\left(Q_{0}, \omega=0\right)$ are shown to demonstrate the changes in amplitude with rising temperature [31]. From $320 \mathrm{~K}$ to $400 \mathrm{~K}$ the amplitude changes about twice as much as between $400 \mathrm{~K}$ and $550 \mathrm{~K}$.

\section{RESULTS AND DISCUSSION}

At first we will consider macroscopic transport parameters of liquid rubidium and how the resulting SE relation evolves with temperature. In Fig. 4 the experimental values for the macroscopic shear viscosity $\eta$ are depicted [7]. These data are tabulated in a critical review of all available experimental data. Typical uncertainties for the experimental viscosity in the lower temperature range are given as 3\% [59]. It exhibits a smooth decay with increasing temperature. Our simulated shear viscosity data show a good agreement with the experimental data. The inset plots the slope $\frac{d \eta}{d T}$ of the viscosity which indicates a change in a temperature range around $450 \mathrm{~K}$ with a stronger viscosity decrease below $450 \mathrm{~K}$. It has been noticed that the density in liquid rubidium also changes in a nonlinear way. The temperature dependence of the density is directly related to the thermal expansion coefficient. The thermal expansion coefficient of liquid rubidium shows an identical change with rising temperature at about $450 \mathrm{~K}$ [60]. The shear viscosity $\eta$ can be represented through the stress

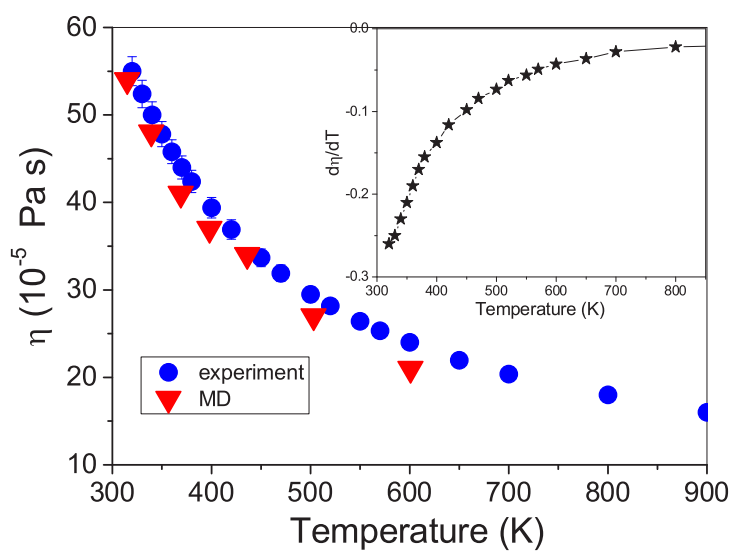

FIG. 4. The experimental shear viscosity $\eta$ (circles) of liquid rubidium is plotted. The inset shows the numerically derived slope of the experimental viscosity data. Included are the results from the viscosity calculation from our MD simulation. 


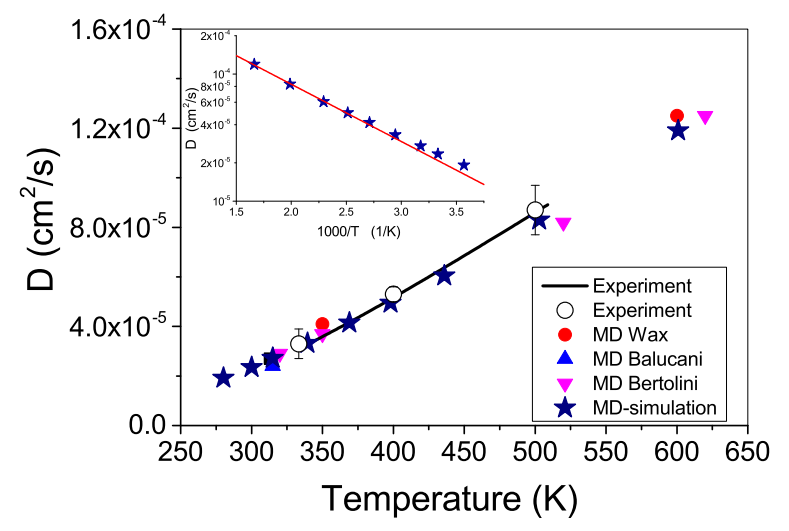

FIG. 5. The figure shows the temperature dependence of the diffusion coefficient $D$ from experiment $[9,61]$ as a line and open symbols. In comparison MD simulation literature results from Balucani et al. (triangles up), Wax et al. (circles), and Bertolini et al. (triangles down) $[32,47,62]$ are added. As stars our diffusion coefficients are included. The inset shows our simulated $D$ values in an Arrhenius-type presentation.

tensor autocorrelation function $[4,15]$ :

$$
\eta=\frac{1}{k_{B} T V} \int_{0}^{\infty}\left\langle\sigma^{x z}(t) \sigma^{x z}(0)\right\rangle d t=\int_{0}^{\infty} \eta(t) d t .
$$

The microscopic stress tensor can be written as

$$
\sigma^{x z}(t)=\sum_{i=1}^{N}\left[m v_{i}^{x}(t) v_{i}^{z}(t)+x_{i}(t) F_{i}^{z}(t)\right] .
$$

Here $F_{i}$ is the total force on atom $i$, which is given by the gradient of the pair potential. The two terms are related to a contribution of the kinetic energy through the displacement of the atoms and one part from the potential energy through the interaction potential. From the microscopic definition it can been seen that the stress tensor describes the flow of momentum in the liquid and hence the viscosity is the Green-Kubo integral of the autocorrelation function of the momentum flux. The correlations of the stress tensor fluctuations decrease strongly in a temperature range up to about $500 \mathrm{~K}$ and then change the gradient. In the same temperature range the correlated fluctuations of volume and entropy, represented through the thermal expansion coefficient, increase strongly [60], and above $500 \mathrm{~K}$ the increase of the thermal expansion coefficient changes distinctly to a quite modest value. Both macroscopic parameters indicate through their changes of fluctuations a change in dynamics in the liquid.

In Fig. 5 experimental and simulation results for the selfdiffusion coefficient $D$ of liquid rubidium are depicted. The experimental data have been obtained by analyzing isotope enrichment through electrotransport [9,61]. The temperature dependence within the experiment has and can be described by an Arrhenius process $D=(6.6 \pm 1.1) \times 10^{-4} \exp (-8290 \pm$ $670 /[8.314 T]) \frac{\mathrm{cm}^{2}}{\mathrm{~s}}$. The relative errors for the diffusion coefficient are therefore in a range of $10-15 \%$. Through the open symbols three experimental data points taken from the publication are denoted [61]. Classical MD simulated data agree quite well with the experimental values $[32,47,62]$. Unfortunately, the experimental data are of limited use concerning

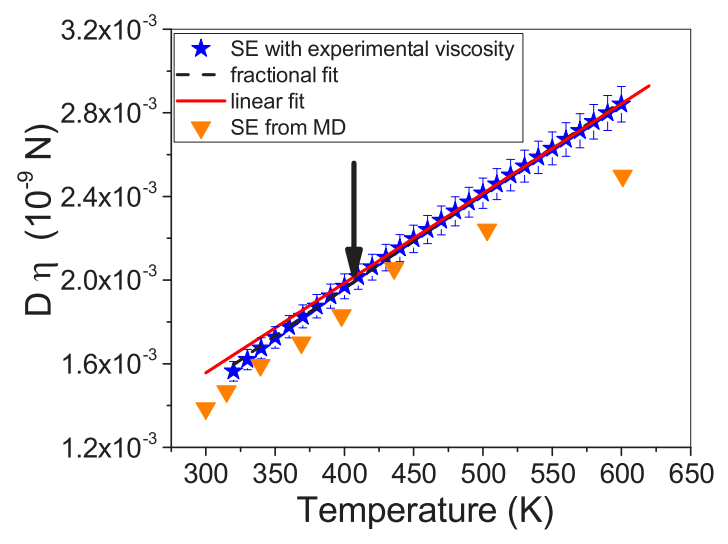

FIG. 6. The SE relation $D \eta$ is plotted over temperature. The hightemperature linear fit is extrapolated to lower temperatures to indicate the $\mathrm{SE}$ relation violation. Included as a dashed line is also a fit with a fractional behavior. The triangles denote $D \eta$ obtained through MD simulation data.

the available temperature range and due to non-negligible error bars. Included in the figure are our results for $D$ from the MD simulation (stars). They agree quite well with previous MD simulation results from different groups with slightly different potential implementations. Also the overall agreement with the experimental values is quite good, which suggests that the self-dynamics is well described by the simulation. Note that the two lower temperatures have been obtained in the supercooled state of rubidium. In the inset we plot the simulation results of $D$ on a logarithmic scale against the inverse temperature. Nachtrieb surveyed a range of liquid metals and concluded that an Arrhenius-type temperature dependence as well as a linear $T$ dependence describe the diffusion coefficients equally well [63]. For an Arrhenius-type behavior one would expect a linear dependence, depicted through the line, which was fitted through the four high-temperature points. Therefore the departure of the low-temperature $D$ values from the expected linear behavior could be regarded as evidence that the dynamics may change in a temperature range around $400 \mathrm{~K}$. On the other side reports exist that diffusion coefficients in liquid metals can be described with a power-law temperature dependence and not with an Arrhenius-type behavior. For example, liquid lead diffusion was described by a $D=A T^{2}$ law [64]. Hence a non-Arrhenius behavior of the diffusion coefficient alone might not indicate a change in the dynamics.

In Fig. 6 the SE relation is presented in the following form: $D \eta \propto T$. Here the tabulated shear viscosity data [7] and the interpolated diffusion coefficients $D$ from our MD simulation are used. The SE relation predicts a linear temperature dependence, which is obviously not fulfilled. The viscosity falls with temperature faster than the diffusion coefficient increases which results in a sublinear increase of the product. The high-temperature points have been fitted with a line which is extended to lower temperatures to demonstrate the deviations from the SE relation within the data. Note that a fit with a fractional temperature behavior $D \eta \propto T^{\gamma}$ with a $\gamma \neq 1$ is able to fit the temperature dependence with a power of 0.92 quite reasonably, although not perfectly (dashed line). The arrow in the figure indicates where the SE relation starts to deviate 


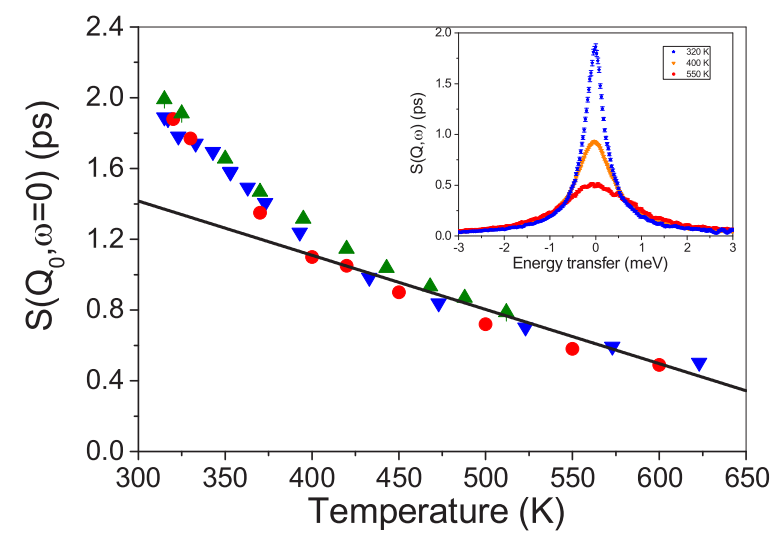

FIG. 7. The amplitude $S\left(Q_{0}, \omega=0\right)$ at the structure factor maximum is plotted over temperature. The different symbols relate to 3 different measurements [26,31]. The error bars are equal to or smaller than the symbol size. The inset shows 3 spectra to demonstrate the changes in amplitude with rising temperature. The line is a fit through the high-temperature points and serves as a guide to the eye.

from the high-temperature expected values. Note a linear fit of the low-temperature values extrapolates to zero value of the SE relation near zero K, whereas the high-temperature linear extrapolated fit deviates from zero value. Included as triangles are the values for the SE relation derived from pure MD simulation data. The MD-data-derived SE relation indicates a strong deviation from the expected linear behavior in a similar temperature range.

To identify changes in the dynamics on an atomic length scale a viable option is to look for density fluctuations at nearest neighbor distances. The slowest dynamics for density fluctuations is expected at the structure factor maximum. This slowing down in liquid dynamics at nearest neighbor distances is well known as de Gennes narrowing [65]. De Gennes derived the frequency moments for the scattering function and found that the normalized second moment is given by $\Omega_{0}^{2}=\frac{k_{B} T Q^{2}}{m S(Q)}$. The moments are a measure of the spread of the spectra and show according to the calculation a decrease of the width when the structure factor $S(Q)$ reaches its maximum, a reflection of how atomistic structure influences the dynamics. In a simple picture, in a dense liquid it costs time for a density fluctuation to relax on a next neighbor length scale due to a necessary rearrangement of the surrounding particles. That momentum transfer is ideal to study structural relaxation on an atomic length scale.

In Fig. 7 the amplitude $S\left(Q_{0}, \omega=0\right)$ is plotted, where $Q_{0}=1.52 \AA^{-1}$ denotes the structure factor maximum of liquid rubidium. We analyzed the amplitude at the structure factor maximum $S\left(Q_{0}, \omega=0\right)$ from three different measurements on liquid rubidium, all three performed with different energy resolution [26,31]. The error bars for determining the amplitudes are smaller than the symbol size. Nevertheless, all data sets agree quite well. The inset illustrates the change of the spectral amplitude with rising temperature. From $320 \mathrm{~K}$ to $400 \mathrm{~K}$ the amplitude changes about twice as much as between $400 \mathrm{~K}$ and $550 \mathrm{~K}$. With increasing temperature the amplitude $S\left(Q_{0}, \omega=0\right)$ decreases in a continuous but nonlinear way. This change was emphasized through a linear fit through the high-temperature points above $400 \mathrm{~K}$. Such a linear fit has no theoretical basis and is merely a guide to the eye. However, there is obviously a change in the slope around $T \approx 400 \mathrm{~K}$. Because we are probing structural relaxations at nearest neighbor distances that change in dynamics is evidence for a change in the local dynamics of density fluctuations. Formally, $S\left(Q_{0}, \omega=0\right)$ is related to the intermediate scattering function $F(Q, t)$ through a Fourier transform:

$$
\begin{aligned}
S\left(Q_{0}, \omega=0\right) & =\left.\frac{1}{2 \pi} \int_{-\infty}^{\infty} F\left(Q_{0}, t\right) \exp (-i \omega t) d t\right|_{\omega=0} \\
& =\frac{1}{\pi} \int_{0}^{\infty} F\left(Q_{0}, t\right) d t .
\end{aligned}
$$

The latter term is the area under the relaxation curve and can be used as a definition for an average relaxation time $\langle\tau\rangle$. An estimate for an average relaxation time $\langle\tau\rangle$ of $F(Q, t)$ can be obtained through [66]

$$
\begin{aligned}
\langle\tau\rangle & =\int_{0}^{\infty} d t \frac{\left\langle n^{*}(Q, t), n(Q, 0)\right\rangle}{\left\langle n^{*}(Q, 0), n(Q, 0)\right\rangle} \\
& =\frac{\int_{0}^{\infty} d t F(Q, t)}{S(Q)}=\frac{\pi S\left(Q_{0}, \omega=0\right)}{S\left(Q_{0}\right)} .
\end{aligned}
$$

For an exponential decay of the intermediate scattering function this expression is exact. Within the glass transition literature a similar expression for a stretched exponential decay has long been used to characterize the relaxation dynamics. The average relaxation times are depicted in Fig. 8(a) on a logarithmic scale versus the inverse temperature. For the temperature-dependent structure factor $S\left(Q_{0}\right)$ interpolated values from the table in [26] have been applied.

If a single relaxation process dominates, then the points should be lying on a single line in this Arrhenius-type plot. The two lines are linear fits of the high and low temperature region to emphasize the changes. As evidenced through the two lines a change in relaxation dynamics occurs in the temperature range of $T \approx 450 \mathrm{~K}$. In Fig. 8 (b) we plot relaxation times obtained from a fit to the $S(Q, \omega)$ spectra. A simple Lorentzian curve was used to fit the data, neglecting further line-shape details near the melting point [31]. At higher temperatures the tails of the spectra are reaching beyond the experimentally available dynamic range of the spectrometers, which might influence the fit results. Both methods deliver similar values for the relaxation of density fluctuations at the structure factor maximum. Also the relaxation times $\tau$ in Fig. 8(b) show a non-Arrhenius behavior with a changing slope around $450 \mathrm{~K}$ (see arrow). Included in the figure are relaxation times of the self-dynamics at the structure factor maximum $\tau_{s}\left(Q_{0}\right)$ and from the collective movements $\tau\left(Q_{0}\right)$ from the MD simulation. The self-intermediate scattering function $F_{s}\left(Q_{0}, t\right)$ and the intermediate scattering function $F\left(Q_{0}, t\right)$ have been fitted by a stretched exponential $F(Q, t)=\exp \left[-(t / \tau)^{\beta}\right]$. The power $\beta$ changes with temperature from 0.8 for the self-dynamics and 0.92 for the collective dynamics at melting temperature to 1 around $400 \mathrm{~K}$. That transformation from a stretched exponential to an exponential decay agrees very well with a previous analysis of experimental $F(Q, t)$ data [31]. The two lowest temperature points are simulated in the supercooled state of rubidium and do not show any evidence of a changing 

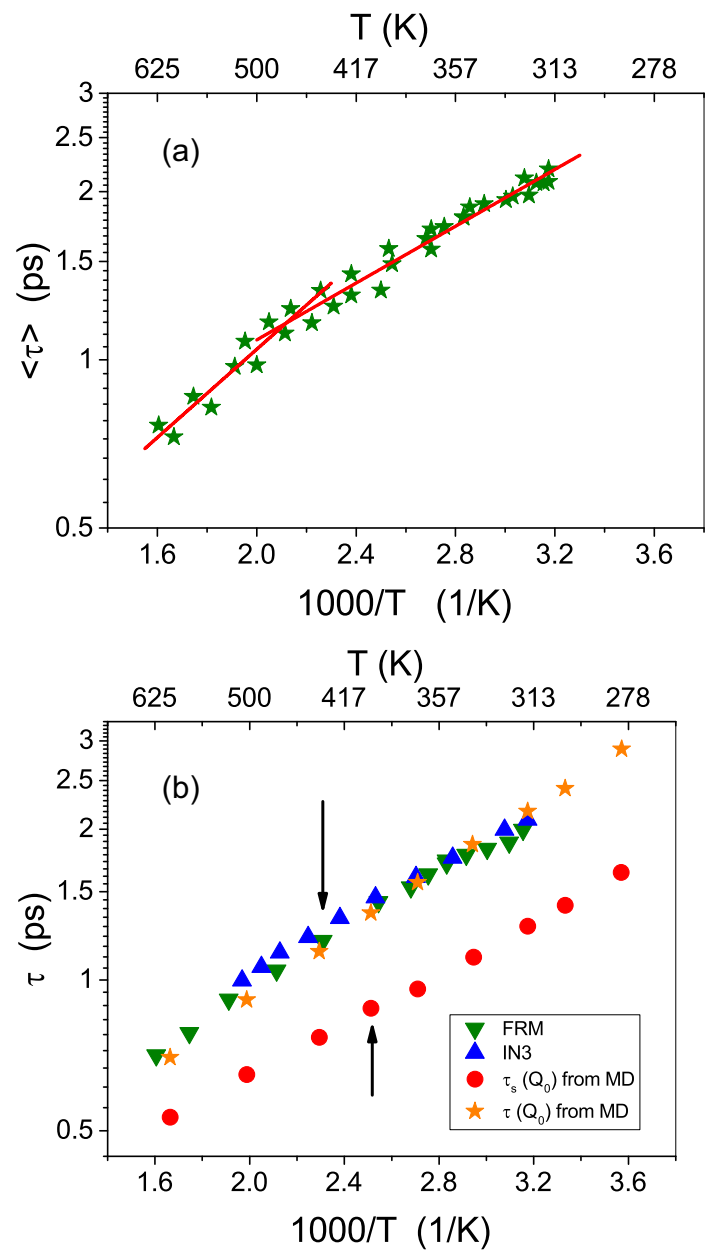

FIG. 8. The logarithm of the average relaxation time $\langle\tau\rangle$ is plotted in panel (a) against the inverse temperature in an Arrhenius-type plot. The straight lines are fits for the respective temperature ranges and are guides for the eye to emphasize the change in dynamics with increasing temperature around $450 \mathrm{~K}$. In panel (b) the relaxation times $\tau\left(Q_{0}\right)$ are plotted extracted from Lorentzian fits to the $S(Q, \omega)$ spectra. Error bars are smaller than symbol size. In addition, the relaxation time $\tau_{s}\left(Q_{0}\right)$ (circles) and $\tau\left(Q_{0}\right)$ (stars) from the self-intermediate correlation function and from the intermediate correlation function from the MD simulation is plotted. Note the lowest two temperatures in the simulation are in the supercooled state. The arrows indicate temperature ranges where the dynamics appear to change.

dynamics when the melting point is crossed. The relaxation times from the self-dynamics indicate only a weak deviation from a linear temperature dependence on this Arrhenius plot (indicated as an arrow). The relaxation times $\tau\left(Q_{0}\right)$ agree well with the experimental data despite the different methods with which they have been obtained. The relaxation times from the simulated intermediate scattering function $F\left(Q_{0}, t\right)$ deviate from a linear Arrhenius behavior and the deviations can be located in the same temperature range where the experimental data show a deviation. The correlated movements, as given through the average relaxation time $\langle\tau\rangle$ and the relaxation times $\tau\left(Q_{0}\right)$, are a much more explicit indicator for the changes in the dynamics than the self-dynamics. The average relaxation time $\langle\tau\rangle$ is identical to our measured amplitude $S\left(Q_{0}, \omega=0\right)$ and can be interpreted as an average decay time of density
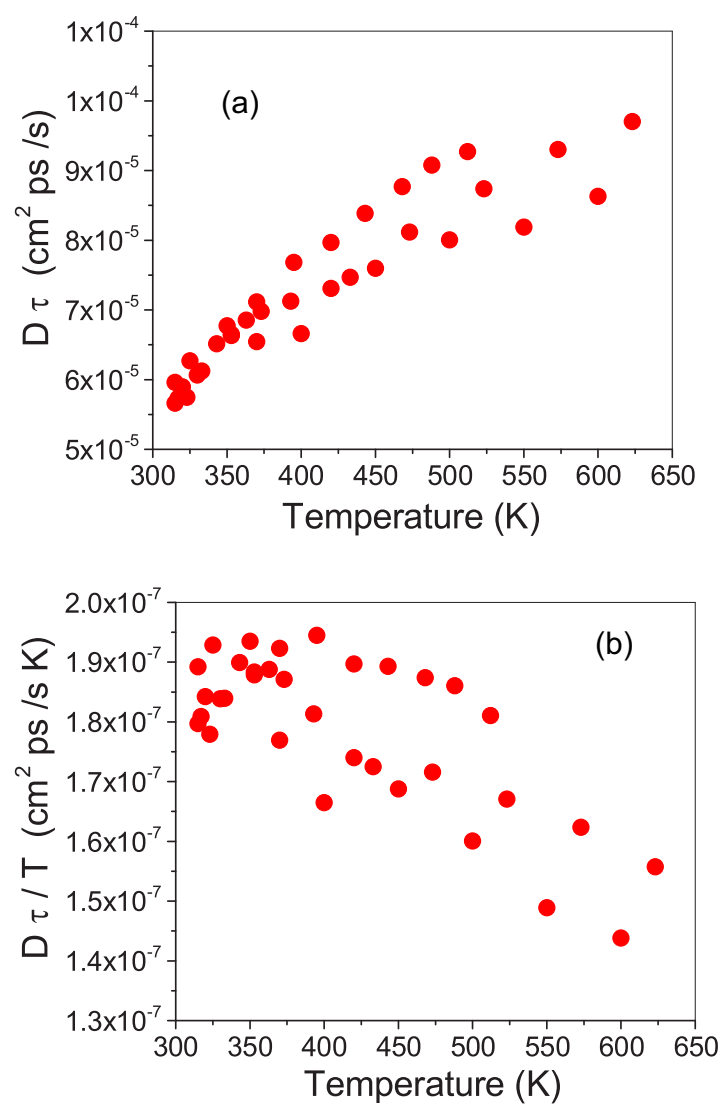

FIG. 9. In panel (a) the SE relation is plotted in the form $D\langle\tau\rangle$ over temperature. A linear temperature dependence is expected, which is clearly violated. In panel (b) the SE relation is plotted in the form $D\langle\tau\rangle / T$ over temperature. A constant value is expected which appears to deviate around $400-450 \mathrm{~K}$.

fluctuations on next neighbor distances. It might be understood as the average time a particle is associated with its nearest neighbors.

Now with access to an average structural relaxation time we can test the second form for the SE relation according to $D \tau \sim T$, relating the macroscopic diffusivity with a microscopic relaxation time. Note that the average relaxation time is obtained from coherent neutron scattering and hence describes correlated particle dynamics as the viscosity does. In Fig. 9(a) the SE relation is plotted according to $D \tau \propto T$. The scatter of the points is related to the variation of the average relaxation times from the three different measurements (see Fig. 7). The expected linear temperature dependence is clearly violated at a temperature of around $400 \mathrm{~K}$. The product of $D \tau$ has units of a length squared and can be regarded as the squared distance a particle moves during the average relaxation time. Near the melting point a particle moves about $0.8 \AA$ during the average relaxation time, which corresponds to about $20 \%$ of a hard sphere diameter of rubidium. That consideration suggests that between melting point and about $400 \mathrm{~K}$ the caged particle increases its mobility within the average relaxation time more than at higher temperatures. In Fig. 9(b) the SE relation is plotted in a different form according to $D \tau / T \propto$ constant. The data points suggest the relation is constant up to a temperature range of about $400-450 \mathrm{~K}$. At higher temperatures 
the data points deviate from the expected constant value and demonstrate the breakdown of the SE relation.

All forms of the SE relation demonstrate a violation with increasing temperature in the equilibrium liquid state of rubidium. The first form is based on experimental macroscopic transport parameters and exhibits an anomalous behavior around $400 \mathrm{~K}$ (see Fig. 6). The second and third forms combine macroscopic diffusion coefficients with a microscopic structural relaxation time and show a violation around $450 \mathrm{~K}$ (see Fig. 9). All forms combine a single particle quantity, the diffusion coefficient, with a quantity related to correlated movements of particles, in one case the macroscopic shear viscosity and in the second case an average relaxation time of density fluctuations on a next neighbor distance. The microscopic relaxation time itself shows a departure from a simple Arrhenius-type behavior. That change in collective dynamics on an atomistic length scale might be the origin for the anomalous behavior of the SE relations.

Further evidences exist for microscopic changes in the dynamics. Previous investigations showed an increase of the generalized longitudinal viscosity in this temperature range upon cooling in experiment and MD simulation [26,32]. That viscosity increase can be understood that liquid rubidium dynamics changes from a high-temperature fluid-like behavior to a more viscous state below that crossover temperature range. One can conclude that the liquid state of liquid rubidium demonstrates distinct changes in the dynamics reflecting a more viscous and a more fluid liquid state. Note that these changes cannot be traced back to the formation of structural motifs, like icosahedrons, inside liquid rubidium [67] and hence must be linked to changes in the dynamics.

Changes in the dynamics above the liquidus temperature in liquid metals and alloys have been reported more recently. With MD simulations on $\mathrm{CuZr}_{2}$ a violation of the $\mathrm{SE}$ relation has been demonstrated at several hundred degrees above the melting point [22]. Similar observations have been reported in a MD simulation of a CuZrAl alloy [24]. Also ab initio based simulations on Al-rich alloys reported a breakdown of the SE relation in the liquid phase [25]. On the experimental side there are not many reports for liquid metals and alloys covering a wide enough temperature range to challenge the SE relation. Brillo et al. reported a violation of the SE relation over a wide temperature range above the melting point [21]. Schober pointed out that collective motions are at the origin of the breakdown of the SE relation there [68]. In a MD simulation study on a $\mathrm{CuZr}$ liquid alloy the observed dynamic heterogeneity was linked to collective stringlike motions and this suggestion supports the view that collective motions are at the center for changes in the dynamics [69]. Our data on the microscopic structural relaxation time of density fluctuations (see Fig. 8) demonstrate that the change in collective dynamics is at the origin of the violation of the SE relation.

Even though the origin of changes in the dynamics might lie with correlated movements in the liquid there will be signs of these changes in the self-dynamics. There is evidence for a change in dynamics in that temperature range from studies on the self-dynamics of a tagged particle [see Fig. 8(b)]. The self-dynamics of the liquid alkali metal sodium has been studied thoroughly from melting point up to the critical point [70]. The dynamics with changing density can be described as

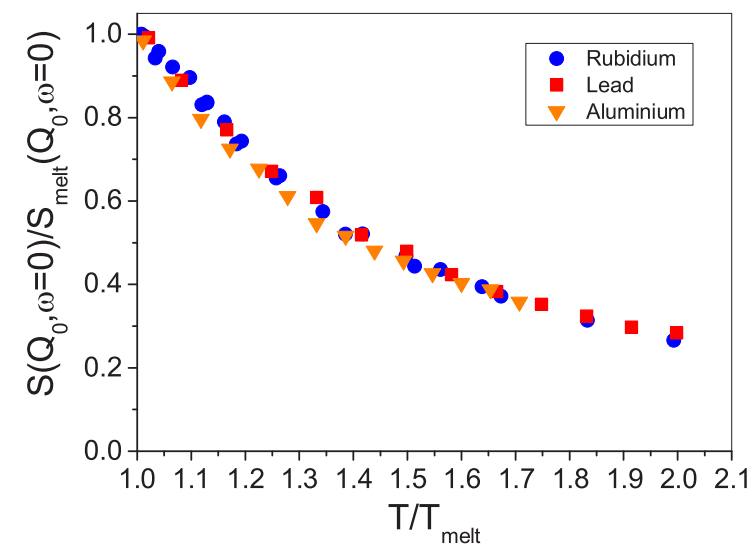

FIG. 10. The normalized amplitudes $S(Q, \omega=0)$ of liquid aluminium, lead, and rubidium are plotted against a normalized temperature.

a competition between mode coupling effects, where near the melting point the coupling to density fluctuations slows down the diffusive process and at high temperature the coupling to transverse excitations increases the diffusion process. A gradual thermal crossover was suggested from a cage-effectdominated regime to one characterized by a vortex pattern around the tagged particle [71]. The crossover between these two processes was estimated to be around $1.3 T_{m}$ [72] for liquid sodium, further evidence that the dynamics in liquid metals change in a temperature region of 1.3-1.5 $T_{m}$.

These reports raise the question of whether the changes in dynamics are a universal feature of metals and alloys. In Fig. 10 we plot the normalized amplitudes $S\left(Q_{0}, \omega=0\right)$ for liquid aluminium, lead, and rubidium. These amplitudes are normalized to their respective value at the melting point and the temperature has also been renormalized to the melting temperature. There is a nearly perfect agreement on how the dynamics at the structure factor maximum changes with rising temperature. All three metals agree on the normalized amplitude value and all three demonstrate a change with rising temperature in the range of $1.3-1.5 T_{m}$. We interpret this agreement as strong support for a universal character of the changes in liquid metal dynamics above the melting temperature.

\section{CONCLUSIONS}

The SE relation of liquid rubidium has been scrutinized over a temperature range between melting point and about twice $T_{m}$ to search for potential violations in the liquid state. Previous studies indicated a change in dynamics from a more viscous liquid metal to a fluid-like liquid state at about $T=1.3-1.5 T_{m}$ in liquid rubidium. To this end an extensive classical MD simulation has been performed over the whole temperature range and also into the undercooled state to complement the available experimental data. From the simulation the selfdynamics has been extracted, which is not accessible from the experiment. The combination of experimental viscosity data and simulated diffusion coefficients, the SE relation, demonstrates a deviation of the expected linear temperature behavior at around $T \approx 400 \mathrm{~K} \approx 1.3 T_{m}$. This observation is based on macroscopic transport parameters. 
On a microscopic scale the amplitude of the dynamic structure factor $S\left(Q_{0}, \omega=0\right)$, related to correlated dynamics on next neighbor distances, changes its slope. From this amplitude an average relaxation time can be derived which could be understood as the mean time a caged particle stays within its environment of next neighbors. The average relaxation time of density fluctuations at this momentum transfer exhibits a non-Arrhenius behavior with a changing slope at around $T \approx 450 \mathrm{~K}$. A SE relation formulated as a product of the single particle diffusion coefficient with that microscopically derived average relaxation time from collective particle movements demonstrates a distinct change at a temperature of about $T \approx 1.3 T_{m}$. These changes signal a transition between different dynamical regimes in liquid rubidium deep within the equilibrium liquid state. Hence, the origin for this crossover in dynamics might be found in changes of the collective dynamics, in particular, in the dynamics on a next neighbor distance.

In the same temperature range a decrease of a generalized longitudinal viscosity was observed upon heating. All these observations can be summarized that liquid rubidium shows two different dynamical regimes, a low-temperature more viscous one and a high-temperature more fluid one separated by a crossover region between $1.3 T_{m}$ and $1.5 T_{m}$. A similar behavior in the amplitude and generalized viscosity was observed in liquid lead and aluminium [27,28]. A comparison of diffusion coefficients for a range of liquid alloys and metals evidences a dynamical crossover at a temperature in the liquid state [73], which might be compatible with the findings here.

After appropriate normalization the amplitudes of liquid aluminium, lead, and rubidium show a remarkable agreement in their temperature dependence and suggest a universal dynamic behavior. Whether such a thermal crossover from a more solid-like to a fluid-like response in the equilibrium liquid state is universal in liquid metals and alloys needs further investigations.

\section{ACKNOWLEDGMENTS}

Gratefully we acknowledge C. Morkel and W. C. Pilgrim for helpful discussions. This work was partially supported by the Science and Technology Facilities Council (STFC).
[1] P. G. Debenedetti and F. H. Stillinger, Nature (London) 410, 259 (2001).

[2] A. Einstein, Ann. Phys. 17, 549 (1905).

[3] L. D. Landau and E. M. Lifshitz, Fluid Mechanics (Pergamon Press, New York, 1959).

[4] J. P. Hansen and I. R. McDonald, Theory of Simple Liquids (Academic Press, London, 2006).

[5] R. Mazo, Brownian Motions (Clarendon Press, Oxford, 2001).

[6] M. Cappelezzo, C. A. Capellari, S. H. Pezzin, and L. A. F. Coehlo, J. Chem. Phys. 126, 224516 (2007).

[7] E. E. Shpilrain, K. A. Yakimovich, V. A. Fomin, S. N. Skovorodjko, and A. G. Mozgovoi, in Handbook of Thermodynamic and Transport Properties of Alkali Metals, edited by R. W. Ohse (Blackwell Scientific Publications, Oxford, 1985).

[8] J. J. van Loef, Physica 75, 115 (1974).

[9] M. Gerl and A. Bruson, in Handbook of Thermodynamic and Transport Properties of Alkali Metals, edited by R. W. Ohse (Blackwell Scientific Publications, Oxford, 1985).

[10] R. Zwanzig, J. Chem. Phys. 79, 4507 (1983).

[11] R. Zwanzig and M. Bixon, Phys. Rev. A 2, 2005 (1970).

[12] U. Balucani, G. Nowotny, and G. Kahl, J. Phys.: Condens. Matter 9, 3371 (1997).

[13] U. Balucani and M. Zoppi, Dynamics of the Liquid State (Clarendon Press, Oxford, 1994).

[14] Z. Shi, P. G. Debendetti, and F. H. Stillinger, J. Chem. Phys. 138, 12A526 (2013).

[15] U. Balucani, R. Vallauri, and T. Gaskell, Phys. Rev. A 37, 3386 (1988).

[16] K. R. Harris, J. Chem. Phys. 131, 054503 (2009).

[17] S. R. Becker, P. H. Poole, and F. W. Starr, Phys. Rev. Lett. 97, 055901 (2006).

[18] L. Xu, F. Mallamace, Z. Yan, F. W. Starr, S. Buldyrev, and H. E. Stanley, Nat. Phys. 5, 565 (2009).

[19] J. A. Hodgdon and F. Stillinger, Phys. Rev. E 48, 207 (1993).

[20] L. Berthier and G. Biroli, Rev. Mod. Phys. 83, 587 (2011).
[21] J. Brillo, A. I. Pommrich, and A. Meyer, Phys. Rev. Lett. 107, 165902 (2011).

[22] X. J. Han and H. R. Schober, Phys. Rev. B 83, 224201 (2011).

[23] X. J. Han, J. G. Li, and H. R. Schober, J. Chem. Phys. 144, 124505 (2016).

[24] A. Jaiswal, T. Egami, and Y. Zhang, Phys. Rev. B 91, 134204 (2015).

[25] N. Jakse and A. Pasturel, Phys. Rev. B 94, 224201 (2016).

[26] F. Demmel, A. Diepold, H. Aschauer, and C. Morkel, Phys. Rev. B 73, 104207 (2006).

[27] F. Demmel, W. S. Howells, and C. Morkel, J. Phys.: Condens. Matter 20, 205106 (2008); F. Demmel, W. S. Howells, C. Morkel, and W. C. Pilgrim, Z. Phys. Chem. 224, 83 (2010).

[28] F. Demmel, A. Fraile, D. Szubrin, W. C. Pilgrim, and C. Morkel, J. Phys.: Condens. Matter 27, 455102 (2015).

[29] U. Balucani and R. Vallauri, Phys. Rev. A 40, 2796 (1989).

[30] F. Demmel, P. Fouquet, W. Häussler, and C. Morkel, Phys. Rev. E 73, 032202 (2006).

[31] F. Demmel and C. Morkel, Phys. Rev. E 85, 051204 (2012).

[32] D. Bertolini, F. Demmel, and A. Tani, Phys. Rev. B 76, 094204 (2007).

[33] L. Köster, K. Knopf, and W. Waschkowski, Z. Phys. A 301, 215 (1981).

[34] J. R. D. Copley and J. M. Rowe, Phys. Rev. Lett. 32, 49 (1974).

[35] R. Winter, W. C. Pilgrim, F. Hensel, C. Morkel, and W. Gläser, J. Non-Cryst. Solids 156-158, 9 (1993).

[36] P. Chieux, J. Dupuy-Philon, J. Jal, and J. B. Suck, J. Non-Cryst. Solids 205-207, 370 (1996).

[37] W. C. Pilgrim, M. Ross, L. H. Yang, and F. Hensel, Phys. Rev. Lett. 78, 3685 (1997).

[38] G. Pratesi, J. B. Suck, and P. A. Egelstaff, J. Non-Cryst. Solids 250-252, 91 (1999). 
[39] F. Demmel, D. Pasqualini, and C. Morkel, Phys. Rev. B 74, 184207 (2006); F. Demmel, A. Diepold, H. Aschauer, and C. Morkel, J. Non-Cryst. Solids 353, 3164 (2007).

[40] J. Bosse, W. Götze, and M. Lücke, Phys. Rev. A 18, 1176 (1978).

[41] L. Sjögren, Phys. Rev. A 22, 2866 (1980).

[42] A. Rahman, Phys. Rev. Lett. 32, 52 (1974).

[43] R. D. Mountain, Phys. Rev. A 26, 2859 (1982).

[44] U. Balucani, R. Vallauri, T. Gaskell, and M. Gori, Phys. Lett. A 102, 109 (1984); U. Balucani, R. Vallauri, and T. Gaskell, Phys. Rev. A 35, 4263 (1987).

[45] G. Kahl and S. Kambayashi, J. Phys.: Condens. Matter 6, 10897 (1994).

[46] D. Pasqualini, R. Vallauri, F. Demmel, Chr. Morkel, and U. Balucani, J. Non-Cryst. Solids 250-252, 76 (1999).

[47] J. F. Wax, R. Albaki, and J. L. Bretonnet, Phys. Rev. B 65, 014301 (2001).

[48] F. Shimojo, Y. Zempo, K. Hoshino, and M. Watabe, Phys. Rev. B 52, 9320 (1995); S. Munejiri, F. Shimojo, and K. Hoshino, J. Phys.: Condens Matter 12, 4313 (2000).

[49] M. M. G. Alemany, J. L. Martins, and B. J. C. Cabral, J. NonCryst. Solids 347, 100 (2004).

[50] A. Kietzmann, R. Redmer, F. Hensel, M. P. Desjarlais, and T. R. Mattsson, J. Phys.: Condens Matter 18, 5597 (2006).

[51] R. D. Murphy and M. L. Klein, Phys. Rev. A 8, 2640 (1973).

[52] M. Silbert, in Liquid Metals, edited by R. Evans and D. A. Greenwood, Institute of Physics Conference Series, Vol. 30 (Institute of Physics, Bristol, 1977), p. 72.

[53] D. L. Price, Phys. Rev. A 4, 358 (1971).

[54] D. L. Price, K. S. Singwi, and M. P. Tosi, Phys. Rev. B 2, 2983 (1970).

[55] W. A. Arrison, Pseudopotentials in the Theory of Metals (Benjamin, New York, 1966).
[56] N. W. Ashcroft, Phys. Lett. 23, 48 (1966).

[57] K. Singwi, A. Sjölander, M. P. Tosi, and R. H. Land, Phys. Rev. B 1, 1044 (1970).

[58] F. Demmel, A. Fleischmann, and W. Gläser, Nucl. Instrum. Methods Phys. Res. Sect. A 416, 115 (1998).

[59] W. D. Weatherford, J. Robert, K. Johnston, and M. L. Valtierra, J. Chem. Eng. Data. 9, 520 (1964).

[60] F. Demmel and C. Morkel, EPJ Web Conf. 151, 02002 (2017).

[61] S. J. Larsson, C. Roxbergh, and A. Lodding, Phys. Chem. Liq. 3, 137 (1972).

[62] U. Balucani, A. Torcini, and R. Vallauri, Phys. Rev. A 46, 2159 (1992).

[63] N. N. Nachtrieb, Adv. Phys. 16, 309 (1967).

[64] G. Mathiak, A. Griesche, K. H. Kraatz, and G. Frohberg, J. NonCryst. Solids 205-207, 412 (1996).

[65] P. G. de Gennes, Physica 25, 825 (1959).

[66] B. J. Berne and R. Pecora, Dynamic Light Scattering (Dover Publications, New York, 2000), p. 15.

[67] F. Demmel and C. Morkel, Phys. Chem. Liq. 50, 31 (2012).

[68] H. R. Schober, Physics 4, 80 (2011).

[69] H. Zhang, C. Zhong, J. F. Douglas, X. Wang, Q. Cao, D. Zhang, and J. Jiang, J. Chem. Phys. 142, 164506 (2015).

[70] W.-C. Pilgrim and C. Morkel, J. Phys.: Condens. Matter 18, R585 (2006).

[71] U. Balucani, A. Torcini, A. Stangl, and Chr. Morkel, J. NonCryst. Solids 205-207, 299 (1996).

[72] A. Stangl, Chr. Morkel, U. Balucani, and A. Torcini, J. NonCryst. Solids 205-207, 402 (1996).

[73] A. Jaiswal, T. Egami, K. F. Kelton, K. S. Schweizer, and Y. Zhang, Phys. Rev. Lett. 117, 205701 (2016). 